\title{
The Muon g-2 in two-Higgs-doublet models
}

\author{
Eung Jin Chun ${ }^{1, a}$ \\ ${ }^{1}$ Korea Institute for Advanced Study, Seoul 130-722, Korea
}

\begin{abstract}
Updating various theoretical and experimental constraints on the four different types of two-Higgsdoublet models (2HDMs), we find that only the "lepton-specific" (or "type X") 2HDM can explain the present muon g-2 anomaly in the parameter region of large $\tan \beta$, a light CP-odd boson, and heavier CP-even and charged bosons which are almost degenerate. The severe constraints on the models come mainly from the consideration of vacuum stability and perturbativity, the electroweak precision data, the $b$-quark observables like $B_{S} \rightarrow \mu \mu$, the precision measurements of the lepton universality as well as the $125 \mathrm{GeV}$ boson property observed at the LHC.
\end{abstract}

\section{Outline}

Since the first measurement of the muon anomalous magnetic moment $a_{\mu}=(g-2)_{\mu} / 2$ by the E821 experiment at BNL in 2001 [1], much progress has been made in both experimental and theoretical sides to reduce the uncertainties by a factor of two or so establishing a consistent $3 \sigma$ discrepancy

$$
\Delta a_{\mu} \equiv a_{\mu}^{\mathrm{EXP}}-a_{\mu}^{\mathrm{SM}}=+262(85) \times 10^{-11}
$$

which is in a good agreement with the different group's determinations. Followed by the 2001 announcement, there have been quite a few studies in the context of 2HDMs [2-4], however, restricted mainly to the type I and II models out of four different types of 2HDMs ensuring natural flavour conservation. Considering the recent experimental development confirming more precisely the Standard Model (SM) predictions, including the discovery of the $125 \mathrm{GeV}$ Brout-Englert-Higgs boson, it would be timely to revisit the issue of the muon $\mathrm{g}-2$ in $2 \mathrm{HDMs}$.

An additional contribution to the muon $\mathrm{g}-2$ from an extra boson in 2HDMs, shown in Fig. 1, may be the origin of the positive excess in the $\Delta a_{\mu}$. This can happen in the type II or X (lepton-specific) 2HDM which allows a light boson having large Yukawa couplings enhanced by $\tan \beta$. While the type II option is completely ruled out by now, the type $\mathrm{X}$ model [5] remains an unique option to explain the $a_{\mu}$ anomaly evading all the recent experimental constraints [6-9].

The previous studies on the muon $\mathrm{g}-2$ in the type II 2HDM (2HDM-II) and various experimental constraints were nicely summarised in Ref. [3]:

- The one-loop correction mediated by a light CP-even (CP-odd and charged) boson gives a positive (negative) contribution to $\Delta a_{\mu}$, and thus the current $3 \sigma$ deviation

\footnotetext{
a e-mail: ejchun@kias.re.kr
}
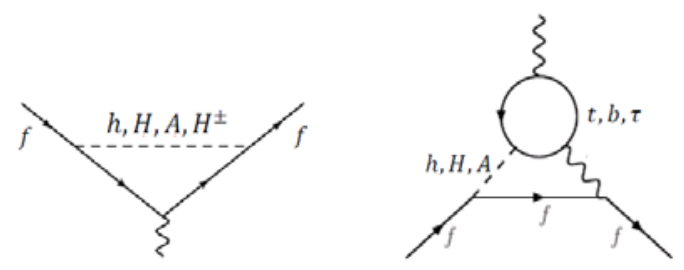

Figure 1. One and two loop diagrams contributing to the muon $\mathrm{g}-2$ in $2 \mathrm{HDMs}$.

can be explained by a CP-even boson lighter than about $5 \mathrm{GeV}$. However, such a light boson was already in contradiction to non-observation of radiative $\Upsilon$ decays $\Upsilon \rightarrow \gamma+X$.

- Contrary to the one-loop correction, the major two-loop contribution from the Barr-Zee diagram [10] mediated by a light $\mathrm{CP}$ odd (even) boson can give a sizable positive (negative) contribution to $\Delta a_{\mu}$. Thus, a light CP odd boson $A$ with large $\tan \beta$ can account for the muon $\mathrm{g}-2$ deviation. However, most of the muon $\mathrm{g}-2$ favoured region in the lower and upper $M_{A}$ part are excluded by the LEP and TEVATRON search on $Z \rightarrow b \bar{b} A(b \bar{b})$, respectively, except a small gap of $M_{A} \approx 25-70 \mathrm{GeV}$ with $\tan \beta \gtrsim 20$.

- However, let us note that such a light $A$ gives a huge contribution to $B_{s} \rightarrow \mu^{+} \mu^{-}$as its rate is proportional to $\tan ^{4} \beta / M_{A}^{4}$ [11], and thus the above gap is completely closed now by the LHC measurement which is consistent with the SM prediction [12].

Now the situation can be quite different in the type $\mathrm{X}$ 2HDM (2HDM-X) where all the extra boson couplings to quarks (leptons) are proportional to $\cot \beta(\tan \beta)$. Due to this, the 2HDM-X becomes hadrophobic in the large $\tan \beta$ limit invalidating most hadron-related constraints applied 
to the type II model. On the other hand, its leptophilic property brings severe constraints from the precision leptonic observables. The key features in confronting the type $\mathrm{X}$ model with the muon $\mathrm{g}-2$ anomaly can be summarized as follows [6-9].

- As in the 2HDM-II, the one-loop correction with a light CP even boson $H$ can account for the muon g-2 excess. While the Upsilon decay suppressed by $1 / \tan ^{2} \beta$ cannot provide a meaningful constraint, the Belle and LHCb searches for $B \rightarrow K \mu \mu$ shut down the muon g-2 favoured region except tiny gaps around $M_{H} \approx 3$ and 4 $\mathrm{GeV}$ [13]. In any case, such a light Higgs boson is excluded by the current measurement of $B_{s} \rightarrow \mu^{+} \mu^{-}$even in the 2HDM-X where $\Gamma\left(B_{s} \rightarrow \mu^{+} \mu^{-}\right) \propto 1 / M_{H}^{4}$.

- The Barr-Zee diagram with the tau lepton in the loop can account for the muon g-2 anomaly again in a parameter region of small $M_{A}$ and large $\tan \beta$ evading all the constraints from the hadron colliders and the $b$-quark observables [6] except the process $B_{s} \rightarrow \mu^{+} \mu^{-}$which rules out $M_{A} \lesssim 10 \mathrm{GeV}$ [7].

- However, the lepton universality test by HFAG [14] combined with the $Z \rightarrow \tau \tau$ decay turns out to limit severely the muon $\mathrm{g}-2$ favoured region of the type $\mathrm{X}$ model [8] allowing (only at $2 \sigma$ ) a small region below $M_{A} \approx 80 \mathrm{GeV}$ and $\tan \beta \approx 60$ [9].

- With such a light $A$, the exotic decay of the $125 \mathrm{GeV}$ boson $h \rightarrow A A$ or $A A^{*}(\tau \tau)$ becomes generically too large unless a certain cancelation is arranged to suppress the $h A A$ coupling $\lambda_{h A A}$ which turns out to be possible only in the wrong-sign limit of the lepton Yukawa coupling [7].

\section{Four types of 2HDMs}

Non-observation of flavour changing neutral currents restricts 2HDMs to four different classes which differ by how the Higgs doublets couple to fermions [16]. They are organized by a discrete symmetry $Z_{2}$ under which different Higgs doublets and fermions carry different parities. These models are labeled as type I, II, "lepton-specific" (or $\mathrm{X}$ ) and "flipped" (or Y). Having two Higgs doublets $\Phi_{1,2}$, the most general $Z_{2}$ symmetric scalar potential takes the form:

$$
\begin{aligned}
V & =m_{11}^{2}\left|\Phi_{1}\right|^{2}+m_{22}^{2}\left|\Phi_{2}\right|^{2}-m_{12}^{2}\left(\Phi_{1}^{\dagger} \Phi_{2}+\Phi_{1} \Phi_{2}^{\dagger}\right) \\
& +\frac{\lambda_{1}}{2}\left|\Phi_{1}\right|^{4}+\frac{\lambda_{2}}{2}\left|\Phi_{2}\right|^{4}+\lambda_{3}\left|\Phi_{1}\right|^{2}\left|\Phi_{2}\right|^{2}+\lambda_{4}\left|\Phi_{1}^{\dagger} \Phi_{2}\right|^{2} \\
& +\frac{\lambda_{5}}{2}\left[\left(\Phi_{1}^{\dagger} \Phi_{2}\right)^{2}+\left(\Phi_{1} \Phi_{2}^{\dagger}\right)^{2}\right]
\end{aligned}
$$

where a (soft) $Z_{2}$ breaking term $m_{12}^{2}$ is introduced. Minimization of the scalar potential determines the vacuum expectation values $\left\langle\Phi_{1,2}^{0}\right\rangle \equiv v_{1,2} / \sqrt{2}$ around which the Higgs doublet fields are expanded as

$$
\Phi_{1,2}=\left[\eta_{1,2}^{+}, \frac{1}{\sqrt{2}}\left(v_{1,2}+\rho_{1,2}+i \eta_{1,2}^{0}\right)\right] .
$$

The model contains the five physical fields in mass eigenstates denoted by $H^{ \pm}, A, H$ and $h$. Assuming negligible CP violation, $H^{ \pm}$and $A$ are given by

$$
H^{ \pm}, A=s_{\beta} \eta_{1}^{ \pm, 0}-c_{\beta} \eta_{2}^{ \pm, 0}
$$

where the angle $\beta$ is determined from $t_{\beta} \equiv \tan \beta=v_{2} / v_{1}$, and their orthogonal combinations are the corresponding Goldstone modes $G^{ \pm, 0}$. The neutral CP-even bosons are diagonalized as

$$
h=c_{\alpha} \rho_{1}-s_{\alpha} \rho_{2}, \quad H=s_{\alpha} \rho_{1}+c_{\alpha} \rho_{2}
$$

where $h(H)$ denotes the lighter (heavier) state.

The gauge couplings of $h$ and $H$ are given schematically by

$$
\mathcal{L}_{\text {gauge }}=g_{V} m_{V}\left(s_{\beta-\alpha} h+c_{\beta-\alpha} H\right) V V
$$

where $V=W^{ \pm}$or $Z$. Taking $h$ as the $125 \mathrm{GeV}$ boson of the $\mathrm{SM}$, the SM limit corresponds to $s_{\beta-\alpha} \rightarrow 1$. Indeed, LHC finds, $c_{\beta-\alpha} \ll 1$ in all the $2 \mathrm{HDMs}$ confirming the SM-like property of the $125 \mathrm{GeV}$ boson [18].

\begin{tabular}{|l|lllllllll|}
\hline & $y_{u}^{A}$ & $y_{d}^{A}$ & $y_{l}^{A}$ & $y_{u}^{H}$ & $y_{d}^{H}$ & $y_{l}^{H}$ & $y_{u}^{h}$ & $y_{d}^{h}$ & $y_{l}^{h}$ \\
\hline Type I & $\cot \beta-\cot \beta-\cot \beta$ & $\frac{\sin \alpha}{\sin \beta}$ & $\frac{\sin \alpha}{\sin \beta}$ & $\frac{\sin \alpha}{\sin \beta}$ & $\frac{\cos \alpha}{\sin \beta}$ & $\frac{\cos \alpha}{\sin \beta}$ & $\frac{\cos \alpha}{\sin \beta}$ \\
Type II & $\cot \beta$ & $\tan \beta$ & $\tan \beta$ & $\frac{\sin \alpha}{\sin \beta}$ & $\frac{\cos \alpha}{\cos \beta}$ & $\frac{\cos \alpha}{\cos \beta}$ & $\frac{\cos \alpha}{\sin \beta}$ & $-\frac{\sin \alpha}{\cos \beta}-\frac{\sin \alpha}{\cos \beta}$ \\
Type X & $\cot \beta-\cot \beta$ & $\tan \beta$ & $\frac{\sin \alpha}{\sin \beta}$ & $\frac{\sin \alpha}{\sin \beta}$ & $\frac{\cos \alpha}{\cos \beta}$ & $\frac{\cos \alpha}{\sin \beta}$ & $\frac{\cos \alpha}{\sin \beta}$ & $-\frac{\sin \alpha}{\cos \beta}$ \\
Type Y & $\cot \beta$ & $\tan \beta-\cot \beta$ & $\frac{\sin \alpha}{\sin \beta}$ & $\frac{\cos \alpha}{\cos \beta}$ & $\frac{\sin \alpha}{\sin \beta}$ & $\frac{\cos \alpha}{\sin \beta}$ & $-\frac{\sin \alpha}{\cos \beta}$ & $\frac{\cos \alpha}{\sin \beta}$ \\
\hline
\end{tabular}

Table 1. The normalized Yukawa couplings for up- and down-type quarks and charged leptons.

Normalizing the Yukawa couplings of the neutral bosons to a fermion $f$ by $m_{f} / v$ where $v=\sqrt{v_{1}^{2}+v_{2}^{2}}=246$ $\mathrm{GeV}$, we have the following Yukawa terms:

$$
\begin{aligned}
& -\mathcal{L}_{\text {Yukawa }}^{2 \mathrm{HDMs}}=\sum_{f=u, d, l} \frac{m_{f}}{v}\left(y_{f}^{h} h \bar{f} f+y_{f}^{H} H \bar{f} f-i y_{f}^{A} A \bar{f} \gamma_{5} f\right)+ \\
& {\left[\sqrt{2} V_{u d} H^{+} \bar{u}\left(\frac{m_{u}}{v} y_{u}^{A} P_{L}+\frac{m_{d}}{v} y_{d}^{A} P_{R}\right) d+\frac{\sqrt{2} m_{l}}{v} y_{l}^{A} H^{+} \bar{v} P_{R} l+h . c .\right.}
\end{aligned}
$$

where the normalized Yukawa couplings $y_{f}^{h, H, A}$ are summarized in Table I for each of these four types of 2HDMs. Let us now recall that the tau Yukawa coupling $y_{\tau} \equiv y_{l}^{h}$ in Type X (also $y_{b} \equiv y_{d}^{h}$ in Type II) can be expressed as

$$
y_{\tau}=-\frac{s_{\alpha}}{c_{\beta}}=s_{\beta-\alpha}-t_{\beta} c_{\beta-\alpha}
$$

which allows us to have the wrong-sign limit $y_{\tau} \sim-1$ compatible with the LHC data [15] if $c_{\beta-\alpha} \sim 2 / t_{\beta}$ for large $t_{\beta} \equiv \tan \beta$ favoured by the muon g-2. Later we will see that a cancellation in $\lambda_{h A A}$ can be arranged only for $y_{\tau}^{h}<-1$ to suppress the $h \rightarrow A A$ decay.

\section{The Muon g-2 from a light CP-odd boson}

Considering all the updated SM calculations of the muon g-2, we obtain

$$
a_{\mu}^{\mathrm{SM}}=116591829(57) \times 10^{-11}
$$



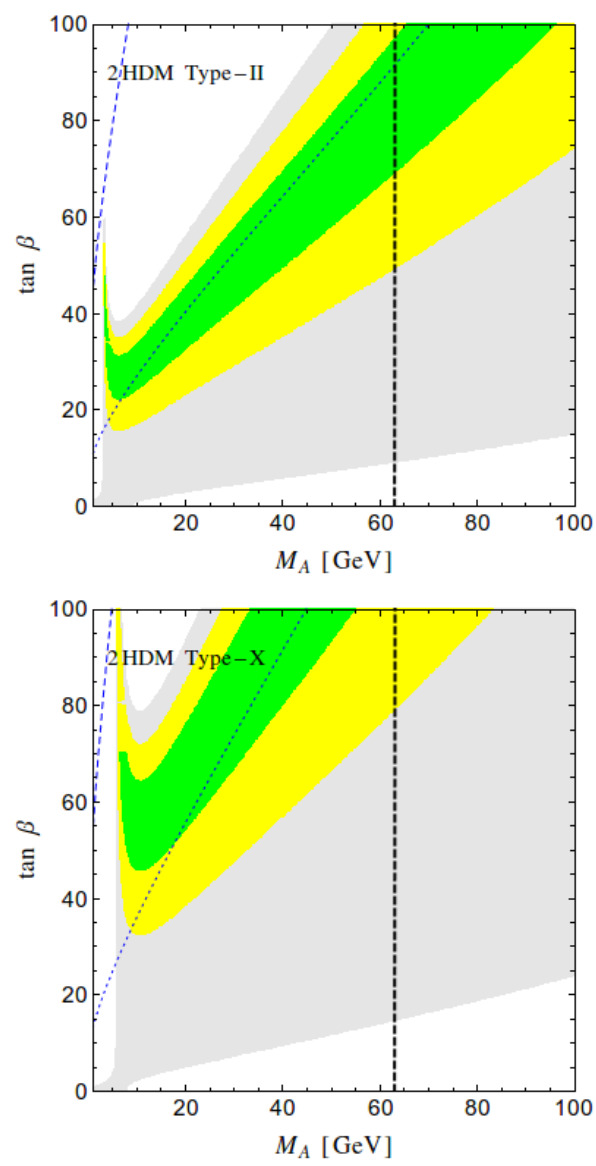

Figure 2. The $1 \sigma, 2 \sigma$ and $3 \sigma$ regions allowed by $\Delta a_{\mu}$ in the $M_{A^{-}}$ $\tan \beta$ plane taking the limit of $c_{\beta-\alpha}=0$ and $M_{h(H)}=125$ (200) $\mathrm{GeV}$ in type II (left panel) and type X (right panel) 2HDMs. The regions below the dashed (dotted) lines are allowed at $3 \sigma(1.4 \sigma)$ by $\Delta a_{e}$. The vertical dashed line corresponds to $M_{A}=M_{h} / 2$.

comparing it with the experimental value $a_{\mu}^{\mathrm{EXP}}=$ $116592091(63) \times 10^{-11}$, one finds a deviation at $3.1 \sigma$ : $\Delta a_{\mu} \equiv a_{\mu}^{\mathrm{EX}}-a_{\mu}^{\mathrm{SM}}=+262(85) \times 10^{-11}$. In the 2HDM, the one-loop contributions to $a_{\mu}$ of the neutral and charged bosons are

$$
\Delta a_{\mu}^{2 \mathrm{HDM}}(1 \text { loop })=\frac{G_{F} m_{\mu}^{2}}{4 \pi^{2} \sqrt{2}} \sum_{j}\left(y_{\mu}^{j}\right)^{2} r_{\mu}^{j} f_{j}\left(r_{\mu}^{j}\right)
$$

where $j=\left\{h, H, A, H^{ \pm}\right\}, r_{\mu}^{j}=m_{\mu}^{2} / M_{j}^{2}$, and

$$
\begin{aligned}
f_{h, H}(r) & =\int_{0}^{1} d x \frac{x^{2}(2-x)}{1-x+r x^{2}}, \\
f_{A}(r) & =\int_{0}^{1} d x \frac{-x^{3}}{1-x+r x^{2}}, \\
f_{H^{ \pm}}(r) & =\int_{0}^{1} d x \frac{-x(1-x)}{1-(1-x) r} .
\end{aligned}
$$

These formula show that the one-loop contributions to $a_{\mu}$ are positive for the neutral scalars $h$ and $H$, and negative for the pseudo-scalar and charged bosons $A$ and $H^{ \pm}$(for
$\left.M_{H^{ \pm}}>m_{\mu}\right)$. In the limit $r \ll 1$,

$$
\begin{aligned}
f_{h, H}(r) & =-\ln r-7 / 6+O(r), \\
f_{A}(r) & =+\ln r+11 / 6+O(r), \\
f_{H^{ \pm}}(r) & =-1 / 6+O(r),
\end{aligned}
$$

showing that in this limit $f_{H^{ \pm}}(r)$ is suppressed with respect to $f_{h, H, A}(r)$. Now the two-loop Barr-Zee type diagrams with effective $h \gamma \gamma, H \gamma \gamma$ or $A \gamma \gamma$ vertices generated by the exchange of heavy fermions gives

$$
\Delta a_{\mu}^{2 \mathrm{HDM}}(2 \mathrm{loop}-\mathrm{BZ})=\frac{G_{F} m_{\mu}^{2}}{4 \pi^{2} \sqrt{2}} \frac{\alpha_{\mathrm{em}}}{\pi} \sum_{i, f} N_{f}^{c} Q_{f}^{2} y_{\mu}^{i} y_{f}^{i} r_{f}^{i} g_{i}\left(r_{f}^{i}\right),
$$

where $i=\{h, H, A\}, r_{f}^{i}=m_{f}^{2} / M_{i}^{2}$, and $m_{f}, Q_{f}$ and $N_{f}^{c}$ are the mass, electric charge and number of color degrees of freedom of the fermion $f$ in the loop. The functions $g_{i}(r)$ are

$$
g_{i}(r)=\int_{0}^{1} d x \frac{\mathcal{N}_{i}(x)}{x(1-x)-r} \ln \frac{x(1-x)}{r},
$$

where $\mathcal{N}_{h, H}(x)=2 x(1-x)-1$ and $\mathcal{N}_{A}(x)=1$.

Note the enhancement factor $m_{f}^{2} / m_{\mu}^{2}$ of the two-loop formula in Eq. (17) relative to the one-loop contribution in Eq. (10), which can overcome the additional loop suppression factor $\alpha / \pi$, and makes the two-loop contributions may become larger than the one-loop ones. Moreover, the signs of the two-loop functions $g_{h, H}$ (negative) and $g_{A}$ (positive) for the $\mathrm{CP}$-even and $\mathrm{CP}$-odd contributions are opposite to those of the functions $f_{h, H}$ (positive) and $f_{A}$ (negative) at one-loop. As a result, for small $M_{A}$ and large $\tan \beta$ in Type II and $\mathrm{X}$, the positive two-loop pseudoscalar contribution can generate a dominant contribution which can account for the observed $\Delta a_{\mu}$ discrepancy. The additional 2HDM contribution $\delta a_{\mu}^{2 \mathrm{HDM}}=\delta a_{\mu}^{2 \mathrm{HDM}}(1$ loop $)+$ $\delta a_{\mu}^{2 \mathrm{HDM}}(2$ loop - BZ) obtained adding Eqs. (10) and (17) (without the $h$ contributions) is compared with $\Delta a_{\mu}$ in Fig. 4.

\section{Electroweak Precision Data}

Allowing such a light CP-odd boson, there could be a strong limit on the extra boson masses coming from the electroweak precision test. To see this, we compare the theoretical 2HDMs predictions for $M_{W}$ and $\sin ^{2} \theta_{\text {eff }}^{\text {lept with }}$ their present experimental values via a combined $\chi^{2}$ analysis. These quantities can be computed perturbatively by means of the following relations

$$
\begin{aligned}
M_{W}^{2} & =\frac{M_{Z}^{2}}{2}\left[1+\sqrt{1-\frac{4 \pi \alpha_{\mathrm{em}}}{\sqrt{2} G_{F} M_{Z}^{2}} \frac{1}{1-\Delta r}}\right] \\
\sin ^{2} \theta_{\mathrm{eff}}^{\text {lept }} & =k_{l}\left(M_{Z}^{2}\right) \sin ^{2} \theta_{W},
\end{aligned}
$$

where $\sin ^{2} \theta_{W}=1-M_{W}^{2} / M_{Z}^{2}$, and $k_{l}\left(q^{2}\right)=1+\Delta k_{l}\left(q^{2}\right)$ is the real part of the vertex form factor $Z \rightarrow l \bar{l}$ evaluated at $q^{2}=M_{Z}^{2}$. We than use the following experimental values:

$$
\begin{aligned}
M_{W}^{\text {EXP }} & =80.385 \pm 0.015 \mathrm{GeV}, \\
\sin ^{2} \theta_{\text {eff }}^{\text {lept,EXP }} & =0.23153 \pm 0.00016
\end{aligned}
$$



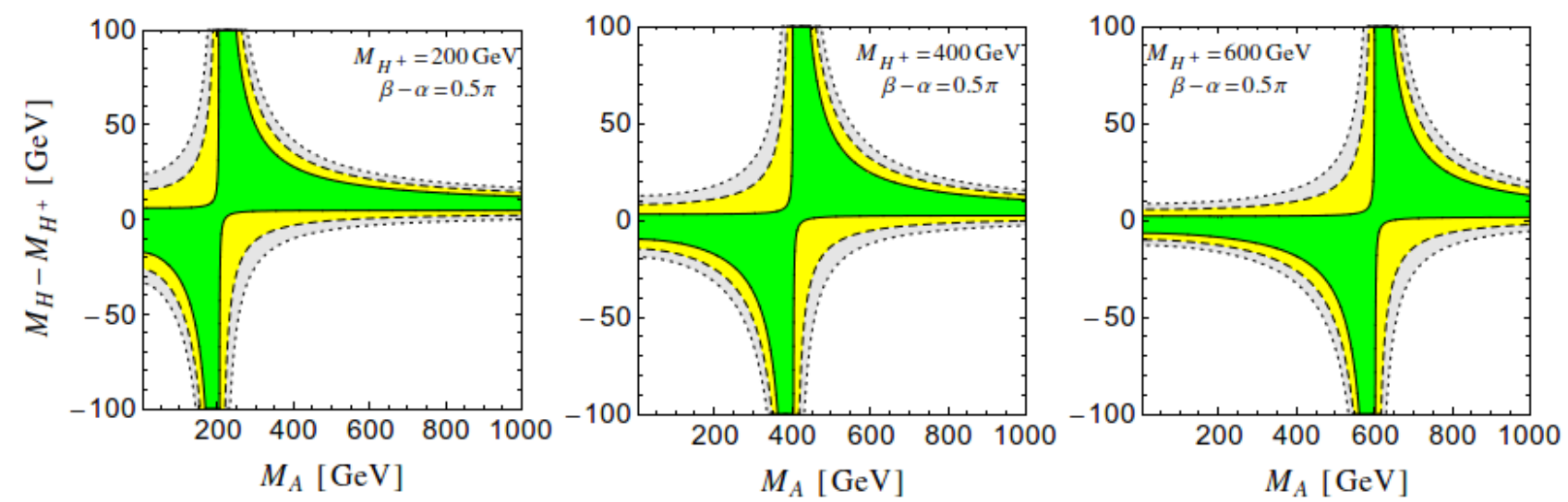

Figure 3. The parameter space allowed in the $M_{A}$ vs. $\Delta M_{H}=M_{H}-M_{H^{ \pm}}$plane by the electroweak precision constraints. The green, yellow, gray regions satisfy $\Delta \chi_{\mathrm{EW}}^{2}\left(M_{A}, \Delta M\right)<2.3,6.2,11.8$, corresponding to $68.3,95.4$, and $99.7 \%$ confidence intervals, respectively.

The results of our analysis are displayed in Fig. 3 confirming a custodial symmetry limit [17] of our interest $M_{A} \ll$ $M_{H} \sim M_{H^{ \pm}}, M_{A} \sim M_{H^{ \pm}} \ll M_{H}$, or $M_{A} \sim M_{H} \sim M_{H^{ \pm}}$ although the last two cases are disfavoured.

\section{Theoretical Consideration of vaccum stability and perturbativity}

While any value of $M_{A}$ is allowed by the EW precision tests in the limit of $M_{H} \sim M_{H^{ \pm}}$, there appear upper bounds on $M_{H^{ \pm}, H}$ by theoretical consideration of vacuum stability, global minimum, and perturbativity expressed respectively by

$$
\begin{aligned}
& \lambda_{1,2}>0, \quad \lambda_{3}>-\sqrt{\lambda_{1} \lambda_{2}}, \quad\left|\lambda_{5}\right|<\lambda_{3}+\lambda_{4}+\sqrt{\lambda_{1} \lambda_{2}}, \\
& m_{12}^{2}\left(m_{11}^{2}-m_{22}^{2} \sqrt{\lambda_{1} / \lambda_{2}}\right)\left(\tan \beta-\left(\lambda_{1} / \lambda_{2}\right)^{1 / 4}\right)>0, \\
& \left|\lambda_{i}\right| \lesssim\left|\lambda_{\max }\right|=\sqrt{4 \pi}, 2 \pi, \text { or } 4 \pi .
\end{aligned}
$$

Taking $\lambda_{1}$ as a free parameter, one can have the following expressions for the other couplings in the large $t_{\beta}$ limit:

$$
\begin{aligned}
& \lambda_{2} v^{2} \approx s_{\beta-\alpha}^{2} M_{h}^{2} \\
& \lambda_{3} v^{2} \approx 2 M_{H^{ \pm}}^{2}-\left(s_{\beta-\alpha}^{2}+s_{\beta-\alpha} y_{\tau}\right) M_{H}^{2}+s_{\beta-\alpha} y_{\tau} M_{h}^{2} \\
& \lambda_{4} v^{2} \approx-2 M_{H^{ \pm}}^{2}+s_{\beta-\alpha}^{2} M_{H}^{2}+M_{A}^{2} \\
& \lambda_{5} v^{2} \approx s_{\beta-\alpha}^{2} M_{H}^{2}-M_{A}^{2}
\end{aligned}
$$

where we have used the relation (8) neglecting the terms of $O\left(1 / t_{\beta}^{2}\right)$.

In the right-sign (RS) limit of the lepton (tau, in particular), $y_{\tau} s_{\beta-\alpha} \rightarrow+1$, one finds a strong upper limit of [6]

$$
M_{A} \ll M_{H^{ \pm}} \sim M_{H} \lesssim 250 \mathrm{GeV} \quad(\mathrm{RS}) .
$$

On the other hand, in the wrong-sign (WS) limit, $y_{\tau} s_{\beta-\alpha} \rightarrow$ -1 , the heavy boson masses up to the perturbativity limit,

$$
M_{A} \ll M_{H^{ \pm}} \sim M_{H} \lesssim \sqrt{4 \pi} v \quad(\mathrm{WS}) .
$$

can be obtained.
Let us finally remark that the $h A A$ coupling is generically order one and thus can lead to a sizeable nonstandard decay of $h \rightarrow A A$ or $A A^{*}(\tau \tau)$ if allowed kinematically. Then, one needs to have $\left|\lambda_{h A A} / v\right| \ll 1$ to avoid an exotic decay of the SM boson. Noting that $\lambda_{h A A} / v \approx$ $s_{\beta-\alpha}\left[\lambda_{3}+\lambda_{4}-\lambda_{5}\right]$, one gets

$$
\lambda_{h A A} v / s_{\beta-\alpha} \approx-\left(1+s_{\beta-\alpha} y_{\tau}\right) M_{H}^{2}+s_{\beta-\alpha} y_{\tau} M_{h}^{2}+2 M_{A}^{2}
$$

where we have put $s_{\beta-\alpha}^{2}=1$. In the RS or SM limit, the condition $\lambda_{h A A} \approx 0$ can be met for a rather light $H$ with $M_{H}^{2} \approx \frac{1}{2} M_{h}^{2}+M_{A}^{2}$ which is disfavoured in the explanation of the muon $\mathrm{g}-2$. On the other hand, one can arrange a cancellation for $\lambda_{h A A} \approx 0$ in the wrong-sign limit for arbitrary value of $M_{H}$ if the tau Yukawa coupling satisfies

$$
y_{\tau} s_{\beta-\alpha} \approx-\frac{M_{H}^{2}-2 M_{A}^{2}}{M_{H}^{2}-M_{h}^{2}}<-1 .
$$

\section{Lepton universality tests}

In the limit of large $\tan \beta$, the charged boson can generate significant corrections to $\tau$ decays at the tree level and furthermore the extra Higgs boson contribution to one-loop corrections can also be significant [19]. The recent study [8] showed that a stringent bound on the charged boson contributions can be obtained from the lepton universality condition obtained by the HFAG collaboration [14]. Given the precision at the level of $0.1 \%$, the lepton universality data put the strongest bound on the type X 2HDM parameter space in favor of the muon g-2. Thus, let us now make a proper analysis of the HFAG data.

From the measurements of the pure leptonic processes, $\tau \rightarrow \mu \nu v, \tau \rightarrow e v v$ and $\mu \rightarrow e v v$, HFAG obtained the constraints on the three coupling ratios, $\left(g_{\tau} / g_{\mu}\right)=$ $\sqrt{\Gamma(\tau \rightarrow e v v) / \Gamma(\mu \rightarrow e v v)}$, etc. Defining $\delta_{l l^{\prime}} \equiv\left(g_{l} / g_{l^{\prime}}\right)-1$, let us rewrite the data from the leptonic processes:

$$
\begin{gathered}
\delta_{\tau \mu}^{l}=0.0011 \pm 0.0015, \\
\delta_{\tau e}^{l}=0.0029 \pm 0.0015, \\
\delta_{\mu e}^{l}=0.0018 \pm 0.0014
\end{gathered}
$$


In addition, combing the semi-hadronic processes $\pi / K \rightarrow$ $\mu \nu$, HFAG also provided the averaged constraint on $\left(g_{\tau} / g_{\mu}\right)$ which is translated into

$$
\delta_{\tau \mu}^{l+\pi+K}=0.0001 \pm 0.0014 .
$$

It is important to notice that only two ratios out of the three leptonic measurements are independent and thus the three data (33) are strongly correlated. For a consistent treatment of the data, one combination out of the three has to be projected out. One can indeed check that the direction $\delta_{\tau \mu}^{l}-\delta_{\tau e}^{l}+\delta_{\mu e}^{l}$ has the zero best-fit value and the zero eigenvalue of the covariance matrix, and thus corresponds to the unphysical direction. Furthermore, two orthogonal directions $\delta_{\tau \mu}^{l}+\delta_{\tau e}^{l}$ and $-\delta_{\tau \mu}^{l}+\delta_{\tau e}^{l}+2 \delta_{\mu e}^{l}$ are found to be uncorrelated in a good approximation. As a result, the 2HDM contribution to $\delta_{l l^{\prime}}$ are calculated to be

$\delta_{\tau \mu}^{l}=\delta_{\text {loop }}, \delta_{\tau e}^{l}=\delta_{\text {tree }}+\delta_{\text {loop }}, \delta_{\mu e}^{l}=\delta_{\text {tree }}, \delta_{\tau \mu}^{l+\pi+K}=\delta_{\text {loop }}$.

Here $\delta_{\text {tree }}$ and $\delta_{\text {loop }}$ are given by [19]:

$\delta_{\text {tree }}=\frac{m_{\tau}^{2} m_{\mu}^{2}}{8 m_{H^{ \pm}}^{4}} \tan ^{4} \beta-\frac{m_{\mu}^{2}}{m_{H^{ \pm}}^{2}} t_{\beta}^{2} \frac{g\left(m_{\mu}^{2} / m_{\tau}^{2}\right)}{f\left(m_{\mu}^{2} / m_{\tau}^{2}\right)}$,

$\delta_{\text {loop }}=\frac{G_{F} m_{\tau}^{2}}{8 \sqrt{2} \pi^{2}} t_{\beta}^{2}\left[1+\frac{1}{4}\left(H\left(x_{A}\right)+s_{\beta-\alpha}^{2} H\left(x_{H}\right)+c_{\beta-\alpha}^{2} H\left(x_{h}\right)\right)\right]$.

where $f(x) \equiv 1-8 x+8 x^{3}-x^{4}-12 x^{2} \ln (x), g(x) \equiv 1+9 x-$ $9 x^{2}-x^{3}+6 x(1+x) \ln (x), H(x) \equiv \ln (x)(1+x) /(1-x)$, and $x_{\phi}=m_{\phi}^{2} / m_{H^{ \pm}}^{2}$. From Eqs. (33), (34) and (35), one obtains the following three independent bounds:

$$
\begin{aligned}
\frac{1}{\sqrt{2}} \delta_{\text {tree }}+\sqrt{2} \delta_{\text {loop }} & =0.0028 \pm 0.0019, \\
\sqrt{\frac{3}{2}} \delta_{\text {tree }} & =0.0022 \pm 0.0017 \\
\delta_{\text {loop }} & =0.0001 \pm 0.0014 .
\end{aligned}
$$

We will use these constraints to put a strong limit on the $(g-2)_{\mu}$ favoured region in the $M_{A}-\tan \beta$ plane in the next section. Let us recall that the $Z \rightarrow \tau \tau$ data, although less strong than the HFAG data, provides an independent bound [8] which further cuts out some corner of parameter space.

\section{Pinning down the whole 2HDM-X parameter space}

It is an interesting task to narrow down the allowed region of the type X 2HDM parameter space collecting all the relevant experimental data including those outlined in Section I and the $125 \mathrm{GeV}$ boson data from LHC, in particular. The scan ranges of all the 2HDM-X input parameters are listed in Table 2. For our scan, we adopt the convention $-\pi / 2<\alpha-\beta<\pi / 2$ and $0<\beta<\pi / 2$, and use the parameter $\lambda_{1}$ as an input parameter instead of $m_{12}^{2}$.

Fig. 4 shows the allowed region in the $m_{A}$ - $\tan \beta$ plane from the profile-likelihood study taking all the other 2HDM-X parameters as nuisance parameters. To see

\begin{tabular}{|c|c|}
\hline 2HDM parameter & Range \\
\hline Scalar boson mass $(\mathrm{GeV})$ & $125<m_{H}<400$ \\
Pseudoscalar boson mass $(\mathrm{GeV})$ & $10<m_{A}<400$ \\
Charged boson mass $(\mathrm{GeV})$ & $94<m_{H^{ \pm}}<400$ \\
$c_{\beta-\alpha}$ & $0.0<c_{\beta-\alpha}<0.1$ \\
$\tan \beta$ & $10<\tan \beta<150$ \\
$\lambda_{1}$ & $0.0<\lambda_{1}<4 \pi$ \\
\hline
\end{tabular}

Table 2. The scan ranges of the 2HDM-X input parameters.

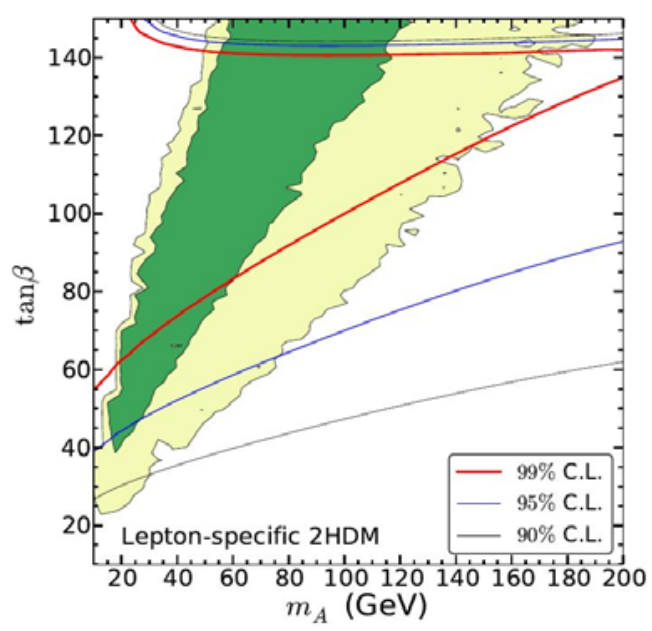

Figure 4. The $68 \%$ and $95 \%$ allowed regions by $\Delta a_{\mu}$ and other experimental constraints in the $m_{A}-\tan \beta$ plane. The contours of the lepton universality likelihood in the $99 \%, 95 \%$ and $90 \%$ confidence level are overlayed.

the impact of the lepton universality data by HFAG, we overlay the contour lines of the lepton universality likelihood at the $99 \%, 95 \%$ and $90 \%$ confidence level based on the constraints (37). The allowed region opened up for $\tan \beta>140$ needs a comment. Note that the $\delta_{\text {loop }}$ is always negative while $\delta_{\text {tree }}$ becomes positive for larger $\tan \beta / m_{H^{ \pm}}$. Thus, there appears a fine-tuned region around $\tan \beta / m_{H^{ \pm}} \sim 1 \mathrm{GeV}^{-1}$ where the positive $\delta_{\text {tree }}$ and the negative $\delta_{\text {loop }}$ cancel each other to give a good fit. However, such regions are excluded by the $Z \rightarrow \tau \tau$ data [8] and thus we are left with the tightly limited region of $M_{A} \approx 10-80$ $\mathrm{GeV}$ and $\tan \beta \approx 25-60$ at the $95 \%$ confidence level.

The region allowed in Fig. 4 can be either in the rightsign $\left(y_{\tau} \equiv \xi_{h}^{l}>0\right)$ or wrong-sign $\left(y_{\tau} \equiv \xi_{h}^{l}<0\right)$ domain as shown in the left and middle panels of Fig. 5. One can see that the rigt-sign limit is tightly constrained to a small region of $m_{A} \approx 60-80 \mathrm{GeV}$ while the wrong-sign limit is favoured in a wider range of parameter space. The right panel shows the sizes of the coupling $\lambda_{h A A}$ restricted by the LHC data on the exotic decay of the $125 \mathrm{GeV}$ boson, putting a generous bound of $\operatorname{Br}\left(h \rightarrow A A^{(*)}\right)<40 \%$. As explained before, the suppressed value of $\lambda_{h A A}$ for $m_{A} \lesssim$ $m_{h} / 2$ is shown to appear only in the wrong-sign domain.

Given the possible existence of a light CP-odd boson explaining the muon g-2 in the type X 2 HDM, it would 

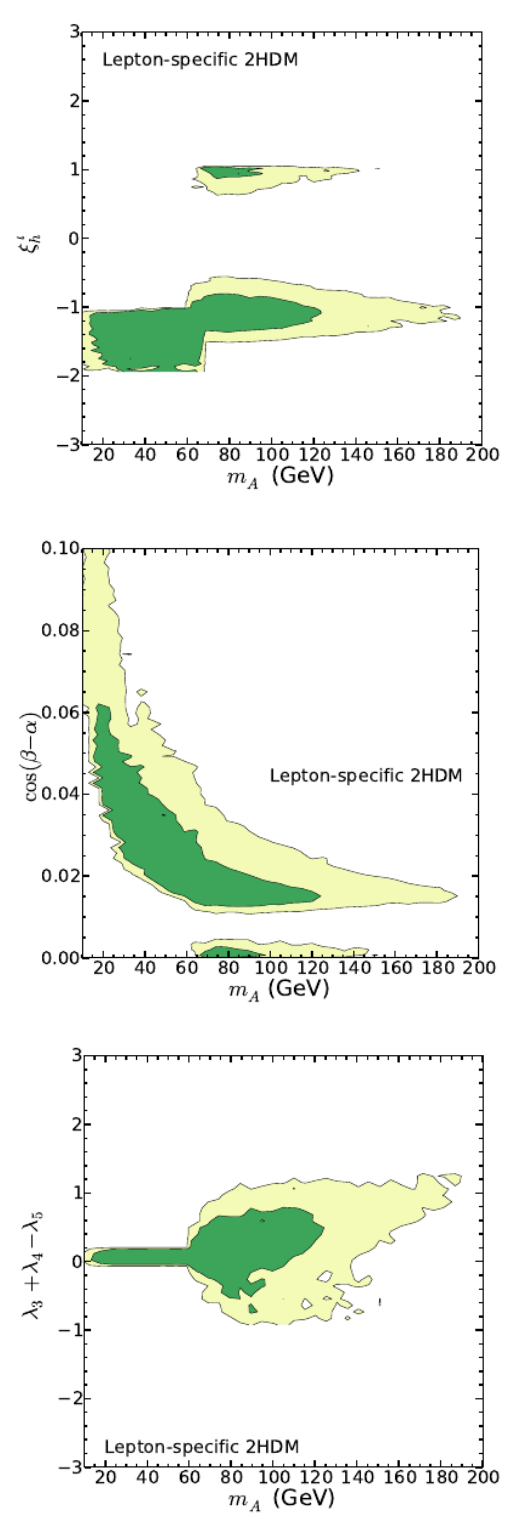

Figure 5. The $68 \%$ and $95 \%$ allowed regions in the $m_{A}-\xi_{h}^{l}\left(y_{\tau} \equiv\right.$ $\left.\xi_{h}^{l}\right)$ (left) and $m_{A}-c_{\beta-\alpha}$ (middle), and $m_{A}-\lambda_{h A A}\left(\lambda_{h A A} \approx \lambda_{3}+\lambda_{4}-\lambda_{5}\right)$ (right) plane.

be important to look for its trails at the LHC. Fig. 6 shows the allowed mass ranges of all the extra bosons. Region A following the pattern of $m_{A} \lesssim m_{H} \approx m_{H^{ \pm}}$is favoured while Region B with $m_{A} \approx m_{H^{ \pm}} \ll m_{H}$ is already excluded as discussed before.

\section{Tau-rich signatures at the LHC}

The bulk parameter space with $m_{A} \ll m_{H} \sim m_{H^{ \pm}}$is a clear prediction of the type X $2 \mathrm{HDM}$ as the origin of the muon g-2 anomaly. Since the extra bosons are mainly from the "leptonic" Higgs doublet with large $\tan \beta$, all the three members are expected to dominantly decay into the $\tau$-flavor, leading to $\tau$-rich signatures at the LHC via the
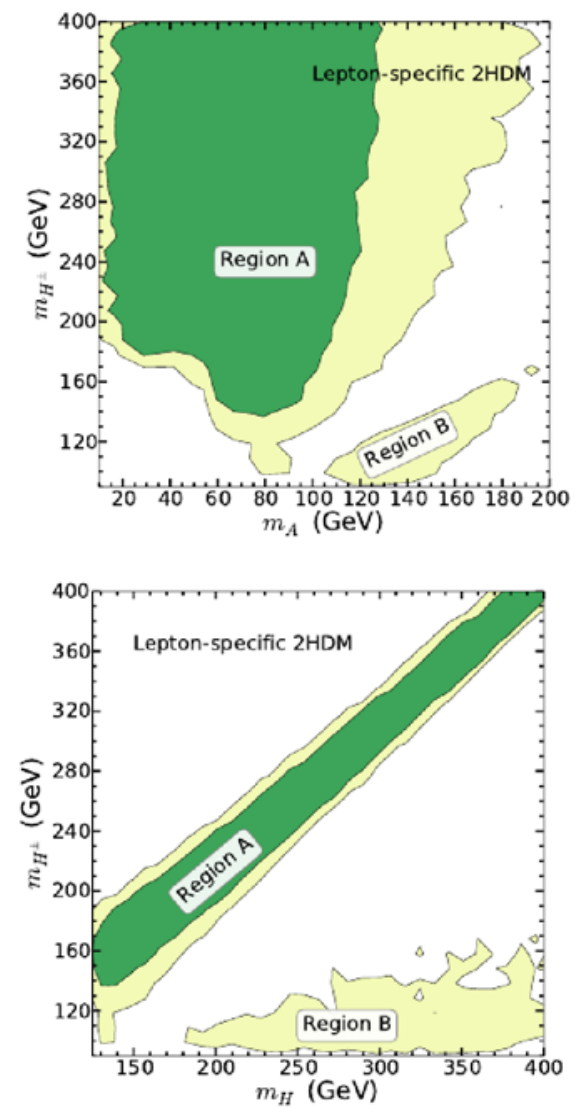

Figure 6. Distribution of the extra boson masses allowed at the $68 \%$ and $95 \%$ confidence levels.

following production and ensuing cascade decay chains:

$$
\begin{aligned}
& p p \rightarrow W^{ \pm *} \rightarrow H^{ \pm} A \rightarrow\left(\tau^{ \pm} v\right)\left(\tau^{+} \tau^{-}\right), \\
& p p \rightarrow Z^{*} / \gamma^{*} \rightarrow H A \rightarrow\left(\tau^{+} \tau^{-}\right)\left(\tau^{+} \tau^{-}\right), \\
& p p \rightarrow W^{ \pm *} \rightarrow H^{ \pm} H \rightarrow\left(\tau^{ \pm} v\right)\left(\tau^{+} \tau^{-}\right), \\
& p p \rightarrow Z^{*} / \gamma^{*} \rightarrow H^{+} H^{-} \rightarrow\left(\tau^{+} v\right)\left(\tau^{-} \bar{v}\right)
\end{aligned}
$$

To probe Region A, we select six benchmark points with different combinations of $m_{A}$ and $m_{H}$ presented in Table 3. For each point, we take a simple parametrization of $\tan \beta=1.25\left(m_{A} / \mathrm{GeV}\right)+25$ and $m_{H^{ \pm}}=m_{H}+15 \mathrm{GeV}$. Note that we included the points with $m_{A}>80 \mathrm{GeV}$ for the sake of the LHC study although they are forbidden by the lepton universality tests. In Table 3 , we show the production cross-section, the selection cuts and the significance for each benchmark expected for the integrated luminosity of $25 / \mathrm{fb}$ at the $14 \mathrm{TeV}$ LHC.

In Fig. 7, we present the exclusion region coming mainly from the chargino-neutralino search at the LHC8, and the expected discovery reaches at LHC14 with the integrated luminosity of $25 / \mathrm{fb}$. A heavy CP-even boson with $m_{H}>200 \mathrm{GeV}$ and a light CP-odd boson with $m_{A}<50 \mathrm{GeV}$ are still allowed, and the LHC14 can explore some of the regions. The sensitivities are weaker for larger $m_{H}$ just because of smaller cross sections, and for smaller $m_{A}$ because $\tau$ s from lighter $A$ become softer and thus the acceptance quickly decreases. Moreover, the 
point $\mathrm{A}$ point $\mathrm{B}$ point $\mathrm{C}$ point $\mathrm{D}$ point $\mathrm{E}$ point $\mathrm{F}$

\begin{tabular}{l|rrrrrr}
\hline$m_{A}[\mathrm{GeV}]$ & 20 & 40 & 100 & 40 & 100 & 180 \\
$m_{H}[\mathrm{GeV}]$ & 200 & 200 & 200 & 260 & 260 & 260 \\
\hline \hline total $\sigma_{\text {gen }}[\mathrm{fb}]$ & 270.980 & 241.830 & 153.580 & 100.430 & 71.271 & 44.163 \\
\hline$n_{\ell} \geq 3$ & 6.606 & 16.681 & 21.713 & 7.110 & 11.962 & 8.822 \\
$n_{\tau} \geq 3$ & 0.894 & 2.602 & 4.386 & 0.888 & 2.346 & 1.971 \\
$H_{T}>100 \mathrm{GeV}$ & 0.201 & 0.547 & 1.179 & 0.209 & 0.765 & 0.926 \\
$n_{b}=n_{j}=0$ & 0.098 & 0.314 & 0.857 & 0.121 & 0.479 & 0.631 \\
\hline \hline$S / B$ & 0.1 & 0.5 & 1.2 & 0.2 & 0.7 & 0.9 \\
$S / \sqrt{B}_{25 \mathrm{fb}^{-1}}$ & 0.6 & 1.9 & 5.2 & 0.7 & 2.9 & 3.8 \\
\hline
\end{tabular}

Table 3. The number of events after applying successive cuts for $14 \mathrm{TeV}$ LHC.
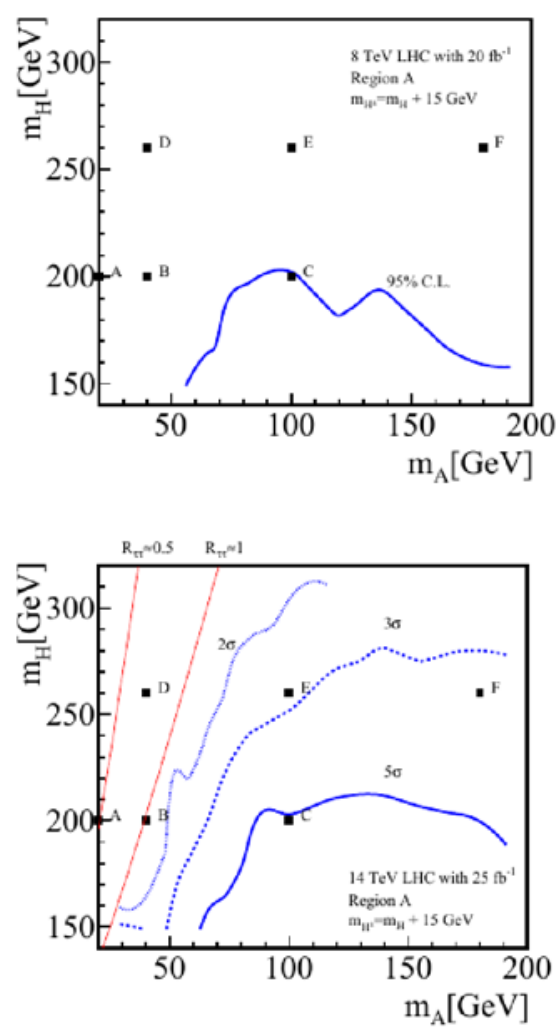

Figure 7. The $95 \%$ exclusion contour from LHC8 (left) and the $2 \sigma, 3 \sigma$ and $5 \sigma$ discovery reach countours at LHC14 (right) in the $m_{A}-m_{H}$ plane.

$H / H^{ \pm} \rightarrow A Z / W^{ \pm}$decay modes also start open to decrease the number of hard $\tau \mathrm{s}$ from direct $H / H^{ \pm}$decays. In such a region, a light $A$ from heavy $H^{+} / H$ decay will be boosted, resulting in a collimated $\tau$-pair which becomes difficult to be tagged as two separated $\tau$-jets. It is one of the reasons to have less acceptance for this parameter region. We can estimate the separation $R_{\tau \tau}$ of the $\tau$ leptons from $A$ decay:

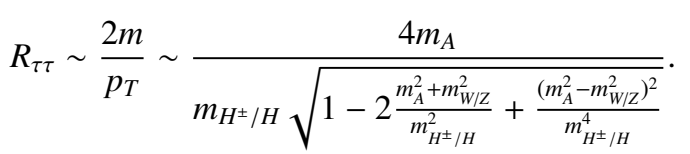

Since the jets are usually defined with $R=0.5$, the $\tau$-pair starts overlapping. We indicated the region with the overlapping $\tau$ problem in red lines in the right panel of Fig. 7.
Further studies on how to capture the kinematic features of the boosted $A \rightarrow \tau^{+} \tau^{-}$are required to probe such a small $m_{A}$ region.

\section{Summary}

The type X 2HDM is still a viable option for the explanation of the muon $\mathrm{g}-2$ in the parameter region with large $\tan \beta$ and a light CP-odd boson $A$. Being "hadrophobic and leptophilic" in the large $\tan \beta$ limit, it can be easily free from all the hadron-related constraints, particularly, coming from the decay $B_{S} \rightarrow \mu \mu$ which puts only a mild bound of $m_{A} \gtrsim 10 \mathrm{GeV}$. However, such a region is tightly limited by the lepton universality tests from the HFAG and $Z \rightarrow \tau \tau$ data. Combining all the current bounds, we find allowed at the $95 \%$ confidence level a limited region of $\tan \beta \approx 15-60$ and $m_{A} \approx 10-80 \mathrm{GeV}$ with $m_{H} \approx m_{H^{ \pm}} \gg m_{A}$.

It will be an interesting task to search for such a light CP-odd boson $A$ and the extra heavy bosons $H, H^{ \pm}$in the next run of the LHC mainly through $p p \rightarrow A H, A H^{ \pm}$followed by the decays $H^{ \pm} \rightarrow \tau^{ \pm} v$ and $A, H \rightarrow \tau^{+} \tau^{-}$which requires further studies to improve the (boosted) tau identification.

\section{References}

[1] H. N. Brown et al. [Muon g-2 Collaboration], Phys. Rev. Lett. 86 (2001) 2227 [hep-ex/0102017]. G. W. Bennett et al. [Muon g-2 Collaboration], Phys. Rev. D 73 (2006) 072003 [hep-ex/0602035].

[2] A. Dedes and H. E. Haber, JHEP 0105 (2001) 006 [hep-ph/0102297]. K. m. Cheung, C. H. Chou and O. C. W. Kong, Phys. Rev. D 64 (2001) 111301 [hep-ph/0103183]. M. Krawczyk, hep-ph/0103223. F. Larios, G. Tavares-Velasco and C. P. Yuan, Phys. Rev. D 64 (2001) 055004 [hep-ph/0103292].

[3] M. Krawczyk, Acta Phys. Polon. B 33 (2002) 2621 [hep-ph/0208076].

[4] K. Cheung and O. C. W. Kong, Phys. Rev. D 68 (2003) 053003 [hep-ph/0302111].

[5] J. Cao, P. Wan, L. Wu and J. M. Yang, Phys. Rev. D 80 (2009) 071701 [arXiv:0909.5148 [hep-ph]].

[6] A. Broggio, E. J. Chun, M. Passera, K. M. Patel and S. K. Vempati, JHEP 1411 (2014) 058 [arXiv:1409.3199 [hep-ph]].

[7] L. Wang and X. F. Han, JHEP 1505 (2015) 039 [arXiv:1412.4874 [hep-ph]].

[8] T. Abe, R. Sato and K. Yagyu, JHEP 1507 (2015) 064 [arXiv:1504.07059 [hep-ph]].

[9] E. J. Chun, Z. Kang, M. Takeuchi and Y.L. Sming Tsai, arXiv:1507.08067, to apear in JHEP.

[10] S. M. Barr and A. Zee, Phys. Rev. Lett. 65 (1990) 21 [Erratum-ibid. 65 (1990) 2920]. V. Ilisie, arXiv:1502.04199 [hep-ph].

[11] H. E. Logan and U. Nierste, Nucl. Phys. B 586 (2000) 39 [hep-ph/0004139].

[12] CMS and LHCb Collaboration, CMS-PAS-BPH-13007. 
[13] K. Schmidt-Hoberg, F. Staub and M. W. Winkler, Phys. Lett. B 727 (2013) 506 [arXiv:1310.6752 [hep$\mathrm{ph}]$ ].

[14] HFAG Collaboration, arXiv:1412.7515 [hep-ph].

[15] P. M. Ferreira, J. F. Gunion, H. E. Haber and R. Santos, Phys. Rev. D 89 (2014) 11, 115003 [arXiv:1403.4736 [hep-ph]]. P. M. Ferreira, R. Guedes, M. O. P. Sampaio and R. San tos, JHEP 1412 (2014) 067 [arXiv:1409.6723 [hep-ph]].

[16] J. F. Gunion and H. E. Haber, Phys. Rev. D 67 (2003) 075019 [hep-ph/0207010]. G. C. Branco,
P. M. Ferreira, L. Lavoura, M. N. Rebelo, M. Sher and J. P. Silva, Phys. Rept. 516 (2012) 1 [arXiv:1106.0034 [hep-ph]].

[17] J.-M. Gerard and M. Herquet, Phys. Rev. Lett. 98 (2007) 251802 [hep-ph/0703051 [HEP-PH]].

[18] ATLAS Collaboration, arXiv:1509.00672; D. Chowdhury and O. Eberhardt, arXiv:1503.08216 [hep-ph].

[19] M. Krawczyk and D. Temes, arXiv:hep-ph/0410348. 